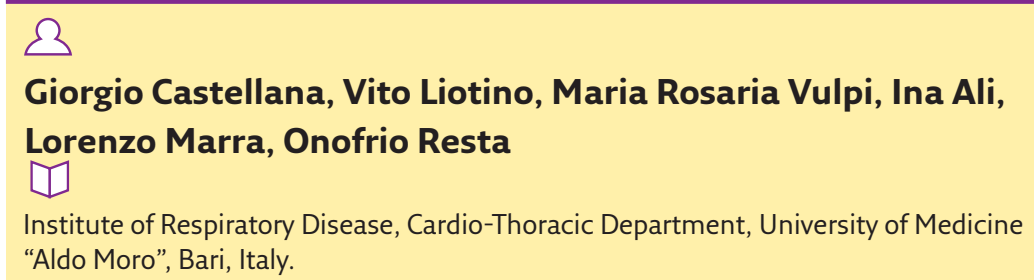

in

https://www.linkedin.com/in/giorgiocastellana-877126a8

\title{
Bilateral pulmonary nodules and acute respiratory failure in a 22-year-old man with dyspnoea and fever
}

\section{Case report}

A 22-year-old white male presented complaining of a 3-week history of fatigue, cough and haemoptysis, accompanied by fever $\left(38^{\circ} \mathrm{C}\right.$ max $)$, dyspnoea and mucopurulent sputum in the last few days. He denied nausea, vomiting, diarrhoea, dysuria and weight loss.

He reported being a smoker (10 pack-years) and a cannabis user, and had a history of allergic rhinitis. He denied occupational exposures.

Before our evaluation, he received empiric antibiotic therapy for 5 days, which resulted in the abatement of fever. Following the persistence of dyspnoea and haemoptysis, he had chest radiography with multiple nodular opacities associated with pleural effusion on the left (figure 1) and lung ultrasound (US) with a convex probe showing multiple hypoechoic, bilateral foci associated with B-lines and bilateral pleural effusion, mainly on the left.

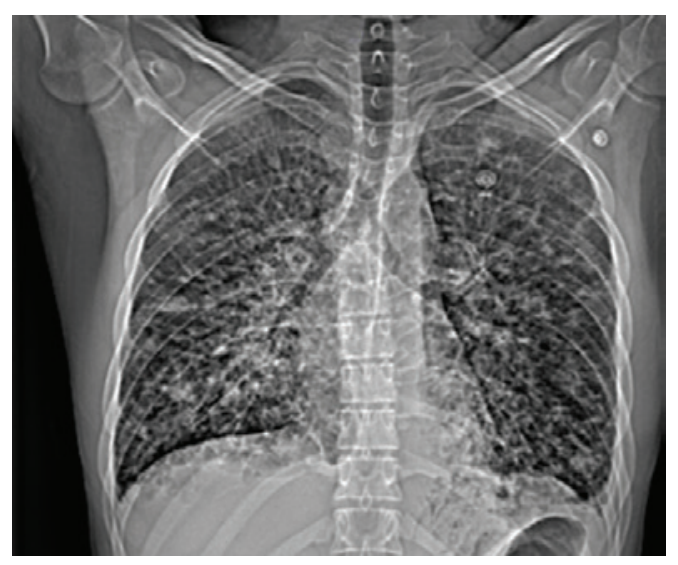

Cite as: Castellana G, Liotino V, Vulpi MR, et al. Bilateral pulmonary nodules and acute respiratory failure in a 22-year-old man with dyspnoea and fever. Breathe 2017; 13: 317-322.
Figure 1 Chest radiograph showing bilateral nodular opacities with a lower zone predominance, interstitial thickening and pleural effusion on the left. 
On our examination, he had no fever. On auscultation, a widespread reduced vesicular murmur and basilar rales in the left lung were found. On palpation, there was inguinal lymphadenopathy. The remainder of the physical examination was unremarkable.

Oxygen saturation by pulse oximetry was $93 \%$ on room air and blood gas analysis showed a mild hypoxaemia, with an arterial oxygen tension $\left(\mathrm{PaO}_{2}\right)$ of $61 \mathrm{mmHg}$ with respiratory alkalosis $(\mathrm{pH} 7.46$, carbon dioxide tension $32 \mathrm{mmHg}$ and bicarbonate $\left.23.2 \mathrm{mmol} \cdot \mathrm{L}^{-1}\right)$, and therefore with a standard $\mathrm{PaO}_{2}$ of $48 \mathrm{mmHg}$.

Laboratory findings were as follows: white blood cell count of 4140 per $\mu \mathrm{L}$ (54.6\% neutrophils, $23.1 \%$ lymphocytes and $20.8 \%$ monocytes), haemoglobin $13.3 \mathrm{~g} \cdot \mathrm{dL}^{-1}$, thrombocytopenia
(123000 per $\mu \mathrm{L}$ ), increased serum inflammatory markers (erythrocyte sedimentation rate $17 \mathrm{~mm} \cdot \mathrm{h}^{-1}$, C-reactive protein $18.4 \mathrm{mg} \cdot \mathrm{L}^{-1}, \alpha_{2}$-globulin $12.8 \%$, $\gamma$-globulin $19.8 \%$, procalcitonin $0.49 \mathrm{ng} \cdot \mathrm{mL}^{-1}$ and presepsin $275 \mathrm{pg} \cdot \mathrm{mL}^{-1}$ ), hypoalbuminaemia (3.1 g. $\mathrm{dL}^{-1}$ ), serum iron and ferritin arrangement of chronic disease (serum iron $63 \mu \mathrm{g} \cdot \mathrm{dL}^{-1}$ and ferritin $553 \mathrm{ng} \cdot \mathrm{dL}^{-1}$ ), slight increase in alanine aminotransferase (48 U. $\left.\mathrm{L}^{-1}\right)$, and direct bilirubin $\left(0.23 \mathrm{mg} \cdot \mathrm{dL}^{-1}\right)$. Renal function and coagulation were in the normal range.

\section{Task 1}

What would be your further investigations for a patient with these features? 


\section{Answer 1}

Total-body computed tomography (CT) in the light of symptoms, of the findings of chest radiography and US, of the inguinal lymphadenopathy and of thrombocytopenia; and microbiological examinations to exclude infectious causes

The patient underwent empirical antibiotic therapy and total-body CT; microbiological tests (of respiratory, blood and urinary samples) were required. Total-body CT showed bilateral pulmonary infiltrate with a pseudonodular aspect, with a tendency toward confluence and surrounded by a ground-glass halo, associated with irregular interlobular interstitial thickening and bilateral pleural effusion. Lymph nodes (LNs) with a colliquative aspect were in the epiaortic, subcarinal, hilar and Barety regions with the maximum diameter of $33 \mathrm{~mm}$ (figure 2). LNs with increased volume were also observed also in the laterocervical, axillary, mesenteric, iliac, lumbar and inguinal regions. There were no abnormalities in the other abdominal and pelvic viscera.
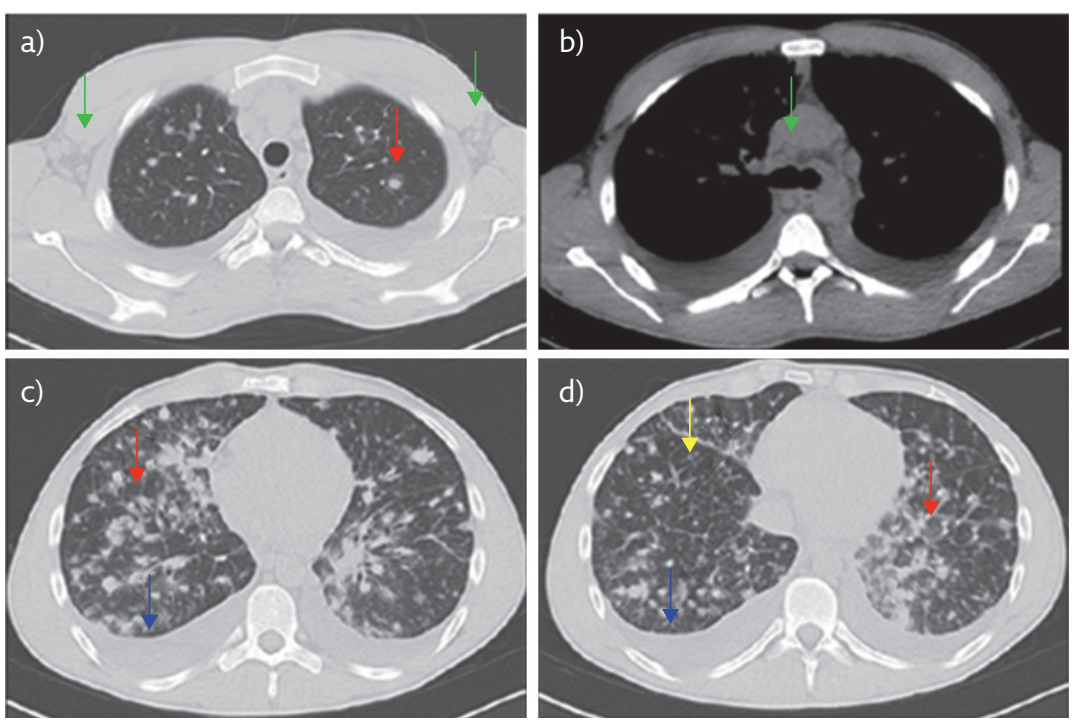

Figure 2 Chest CT (axial view) with parenchymal ( $a, c$ and d) and mediastinal windows (b). a) Upper lung fields: infiltrate with pseudonodular aspect, surrounded by a ground-glass halo (red arrow) and axillar lymph nodes (green arrows). b) Middle lung fields: mediastinal lymph nodes (green arrow). $c$ and d) Lower lung fields: infiltrates with pseudonodular aspect with a tendency toward confluence (red arrows), interlobular interstitial thickening (orange arrow) and pleural effusion (blue arrows).

Task 2

What could be the differential diagnoses in the light of the CT report? 


\section{Answer 2}

Infective disease, lymphoma, metastasis, autoimmune disease or Kaposi's sarcoma (KS)

Bronchoscopy was performed and showed a normal endobronchial mucosa. Biopsy was not performed because of hypoxaemia; however, microbiological and cytological examinations of bronchoalveolar lavage fluid were negative.

Other microbiological examinations searching for common bacteria, Mycobacterium tuberculosis, nontuberculous mycobacteria, viruses and fungi were negative, with the exception of:
- nasopharyngeal swab positive for coronavirus (real-time PCR)

- oral swab positive for Candida albicans

- IgG for Epstein-Barr virus and cytomegalovirus

Tumour and autoimmune (antinuclear antibody and anti-neutrophil cytoplasmic antibody) markers were required.

\section{Task 3}

What would be your next step? 


\section{Answer 3}

Haematological evaluation, HIV test and immune system assessment; US of peripheral lymph nodes in order to detect suspicious lymphadenopathies

Consent for an HIV test was acquired and US examination of superficial LNs followed the haematological evaluation. US showed rounded lymphadenopathy with chaotic vascularisation in the right inguinal and left supraclavicular regions with maximum diameter of $21 \times 13 \mathrm{~mm}$ and $17 \times 10 \mathrm{mmn}$ respectively. Surgical biopsy of the right inguinal lymph nodes was performed and the histological examination result was reported as a morphological aspect consistent with massive metastasis of KS (figure 3). On immunohistochemistry (IHC), diffuse positivity for HHV8 and CD34 was seen in the tumoural area, and that for factor VIII, actin and smooth muscle actin in the rich vasculature. At the same time, an HIV test was positive (HIV-1 gp120, gp41, p24 and p17 were detected), and a T-lymphocyte count in peripheral blood showed no CD4+ cells, and reduced counts of $\mathrm{CD}^{+}$and $\mathrm{CD} 16 / 56^{+} \mathrm{CD}^{-}$cells. Tumour markers and autoimmunity examinations were within normal range, except for CA-125 (71.5 U.mL-1) and $\alpha_{2}$-microglobulin (3.34 $\mathrm{mg} \cdot \mathrm{L}^{-1}$ ). During the hospitalisation, the patient had fever, which was initially intermittent then continuous, accompanied by deterioration of gas exchange, which prompted an increase in the oxygen therapy. In light of this clinical picture, a diagnosis of KS was made.

\section{Discussion}

\section{Definition}

$\mathrm{KS}$ is a slowly growing, multicentric, angioproliferative tumour associated with human herpesvirus (HHV) 8 infection [1]. It is the most common tumour associated with HIV-1 infection; indeed, the incidence among patients infected with HIV is one in 20 [2, 3]. Four clinical variants of KS have been described:

- the classic variant involves elderly men of Mediterranean and Eastern European Jewish origin [4, 5]

- the endemic variant primarily affects men from east and central Africa

- the iatrogenic variant is associated with immunosuppression and tends to be more aggressive

- the AIDS-related variant affects patient with HIV-infection

KS usually presents with cutaneous lesions but it may involve other organs, mostly in the pulmonary and gastrointestinal systems. Intrathoracic

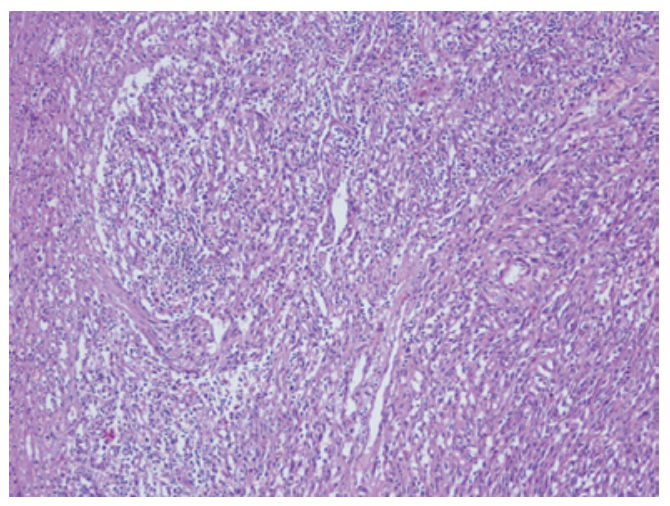

Figure 3 Histological findings from surgical biopsy of the right inguinal lymph nodes: fascicles of monomorphic spindle cells and vascular structure mixed with chronic infiltrates. $100 \times$ magnification.

localisation occurs in $20-75 \%$ of cases, particularly in severely immunocompromised patients with low $\mathrm{CD}^{+}$counts, with involvement of the lung parenchyma, pleura, LNs and airways [6-9]. Reports of isolated pulmonary disease have rarely appeared in the literature $(0-16 \%$ of cases) $[6,7]$.

Pulmonary KS is associated with poor outcome, but highly active antiretroviral therapy has been shown to markedly improve the prognosis.

\section{Clinical, radiological and bronchoscopic characteristics of pulmonary KS}

Common symptoms are dry cough and dyspnoea [6]. Fever and haemoptysis can also be found. Typical radiological features are reticular opacities and parenchymal nodules with a bronchovascular distribution, especially in lower lung zones; consolidation; peribronchial cuffing; pleural effusion; and mediastinal lymphadenopathy [6]. However, chest radiography may be normal. A CT scan is more sensible and specific, and can show irregular, poorly defined nodules with speculated borders and peribronchovascular distribution, interstitial thickening and perinodular ground-glass halo signs (due to haemorrhage secondary to fragility of the neovascular tissue). Irregular narrowing of the bronchial lumen, interlobular septal thickening, air-space consolidation and pleural effusion may be associated with the aforementioned findings [6, 8]. Lymphadenopathy can be present in up to $30-35 \%$ of patients. The effusion can be transudative or exudative and is often haemorrhagic because of the vascular nature of the disease [10].

Typical findings on bronchoscopy are red or purple maculopapular lesions that can narrow and obstruct the entire tracheobronchial tree. The identification of these lesions in the presence of classic radiographic involvement is sufficient to make diagnosis of pulmonary KS in the absence of evidence for opportunistic infections [11].

Biopsy may be needed when parenchymal lesions occur in the absence of endobronchial 
involvement [10]. However, the diagnostic yield of endobronchial biopsies varies between $26 \%$ and $60 \%$ in different studies, and there is high risk of bleeding, especially for centrally located lesions [12, 13]: the decision to obtain a biopsy must be carefully weighed.

\section{Histopathological features}

The histopathology of KS is nearly the same in all variants of disease. Advanced lesions consist of fascicles of spindle cells and vascular structure mixed with chronic infiltrates. On IHC, typical markers are CD31, CD34 and factor VIII-related antigen. Spindle cells express lymphatic markers such as LYVE-1 (lymphatic vessel endothelial hyaluronan receptor-1), VEGFR-3 (vascular endothelial growth factor receptor-3) and Prox-1 (Prospero-related homeobox-1). The most important test to distinguish $\mathrm{KS}$ from others KS-mimicking diseases is the identification of HHV8 within KS lesion cells by immunostaining for LANA-1 (or LNA-1) [10].

\section{Differential diagnosis}

Pulmonary KS is often indistinguishable from other opportunistic lung diseases in patients with AIDS. The differential diagnosis of diffuse pulmonary nodules includes: infective disease (tuberculosis or nontuberculous mycobacterial infection, fungal infection, or septic emboli), neoplastic disease (metastasis, lymphoma or minimally invasive adenocarcinoma), sarcoidosis and other miscellaneous conditions (granulomatosis with polyangiitis, pulmonary alveolar microlithiasis and pulmonary amyloidosis). It is very important to evaluate the size and location of nodules, and the presence of cavities [14-17].

The halo sign has been described in fungal, mycobacterial and viral infections; inflammatory disease (granulomatosis with polyangiitis) and malignancy. In immunocompromised patients, this radiological finding suggests an infection (e.g. invasive aspergillosis) or, less commonly, pulmonary KS and lymphoproliferative disorders [18].

In patients with HIV infection, subcentimetre pulmonary nodules with centrilobular distribution and cavitation may suggest infective disease, whereas larger nodules can suggest malignancy [19]

\section{Conclusion}

Isolated intrathoracic KS is rarely seen. Moreover, in our case, the diagnosis was made more difficult by the lack of the patient's knowledge of his HIV infection. Clinical suspicion, accurate radiological description and multidisciplinary management were decisive in reaching a correct diagnosis.

\section{Conflict of interest}

None declared.

\section{References}

1. Chang Y, Cesarman E, Pessin MS, et al. Identification of herpesvirus-like DNA sequences in AIDS-associated Kaposi's sarcoma. Science 1994; 266: 1865-1869.

2. La Ferla L, Pinzone MR, Nunnari G, et al. Kaposi's sarcoma in HIV-positive patients: the state of art in the HAART-era. Eur Rev Med Pharmacol Sci 2013; 17: 2354-2365.

3. Mbulaiteye SM, Parkin DM, Rabkin CS. Epidemiology of AIDS related malignancies an international perspective. Hemato Oncol Clin North Am 2003; 17: 673-696.

4. Grayson W, Pantanowitz L. Histological variants of cutaneous Kaposi sarcoma. Diagn Pathol 2008; 3: 31.

5. Restrepo CS, Martínez S, Lemos JA, et al. Imaging manifestations of Kaposi sarcoma. Radiographics 2006; 26: 1169-1185.

6. Aboulafia DM. The epidemiologic, pathologic, and clinical features of AIDS-associated pulmonary Kaposi's sarcoma. Chest 2000; 117: 1128-1145.

7. Khalil AM, Carette MF, Cadranel JL, et al. Intrathoracic Kaposi's sarcoma: CT findings. Chest 1995; 108: 1622-1626.

8. Gasparetto TD, Marchiori E, Lourenço S, et al. Pulmonary involvement in Kaposi sarcoma: correlation between imaging and pathology. Orphanet J Rare Dis 2009; 4: 18.

9. Cadranel J, Naccache J, Wislez M, et al. Pulmonary malignancies in the immunocompromised patient. Respiration 1999; 66 289-309.

10. Hashmi HR, Niazi M, Adrish M. A woman in her 30s with a past history of HIV disease presented with recurrent fever, night sweats, and small bilateral pulmonary nodules. Chest 2016; 149: e177-e182
11.Zibrak JD, Silvestri RC, Costello P, et al. Bronchoscopic and radiologic features of Kaposi's sarcoma involving the respiratory system. Chest 1986; 90: 476-479.

12. Meduri GU, Stover DE, Lee M, et al. Pulmonary Kaposi's sarcoma in the acquired immune deficiency syndrome: clinical, radiographic, and pathologic manifestations. Am J Med 1986; 81: 11-18.

13. Baaklini WA, Reinoso MA, Gorin $A B$, et al. Diagnostic yield of fiberoptic bronchoscopy in evaluating solitary pulmonary nodules. Chest 2000; 117: 1049-1054.

14. Boitsios G, Bankier AA, Eisenberg RL. Diffuse pulmonary nodules. AJR Am J Roentgenol 2010; 194: W354-W366.

15. Raoof S, Amchentsev A, Vlahos I, et al. Pictorial essay: multinodular disease: a high-resolution CT scan diagnostic algorithm. Chest 2006; 129: 805-815.

16. Gruden JF, Webb WR, Warnock M. Centrilobular opacities in the lung on high-resolution CT: diagnostic considerations and pathologic correlation. AJR Am J Roentgenol 1994; 162: 569-574.

17. MacMahon H, Naidich DP, Goo JM, et al. Guidelines for management of incidental pulmonary nodules detected on CT images: from the Fleischner Society 2017. Radiology 2017: 284: 228-243

18. Joshi M, Markelova N, Palacio D, et al. A patient with HIV, dyspnea, and multiple pulmonary nodules: pulmonary Kaposi sarcoma. Chest 2006; 130: 1924-1928.

19. Jasmer RM, Gotway MB, Creasman JM, et al. Clinical and radiographic predictors of the etiology of computed tomographydiagnosed intrathoracic lymphadenopathy in HIV-infected patients. J Acquir Immune Defic Syndr 2002; 31: 291-298. 